\title{
Preprint wipes millions off CRISPR companies' stocks
}

In January, the stock of three companies developing therapies based on CRISPR-Cas9 technology fell precipitously. CRISPR Therapeutics, Intellia Therapeutics and Editas Medicine, all based in Cambridge, Massachussetts, roughly lost a combined $\$ 500$ million in market value in a day. The reason? A preprint from the groups of Matthew Porteus and Kenneth Weinberg at Stanford University (https://www. biorxiv.org/content/early/2018/01/05/243345) that suggests preexisting immunity against the Cas9 enzyme may be an issue for gene editing therapies. As Cas endonucleases originate from bacteria, it is not surprising that adaptive immune responses to Cas9 exist in people, but the preprint put immunogenicity front and center as a question for CRISPR-Cas9 companies with products progressing toward clinical applications.

This is not the first time notoriously fickle biotech stocks have fallen (or risen) on the release of scientific findings, but it is the first time a preprint has caused mayhem on the biotech markets, wiping millions of dollars from company valuations and garnering numerous headlines speculating on the future of an experimental therapeutic modality. The hyperbolic media attention given to the preprint has caused consternation in some circles and raises questions for industry and investors about the growing use and dissemination of information from biology preprint servers. Although preprints have long been standard in the physics community, life science researchers have begun to adopt them only recently as an alternative to peer-reviewed publications. There has been little conversation of the implications of how the availability and use of this information by the public and investors might affect biotech companies.

"In this case, the lay press got ahead of the peer-review process," says Amy Wagers, a professor of stem cell and regenerative biology at Harvard University and Harvard Medical School, who states she is generally in favor of preprint servers.

The bioRxiv preprint server that hosts the Porteus and Weinberg paper currently draws the largest number of uploads per month of all those serving the biology community-about 1,000 . Indeed, preprints are increasingly being recognized by biologists as a flexible and rapid way of sharing papers, meeting reports, notices, announcements and data among scientfic peers, compared with peer-reviewed journals, where publication can often take many months. The servers, say proponents, including Stephen Quake, co-president of the Chan Zuckerberg

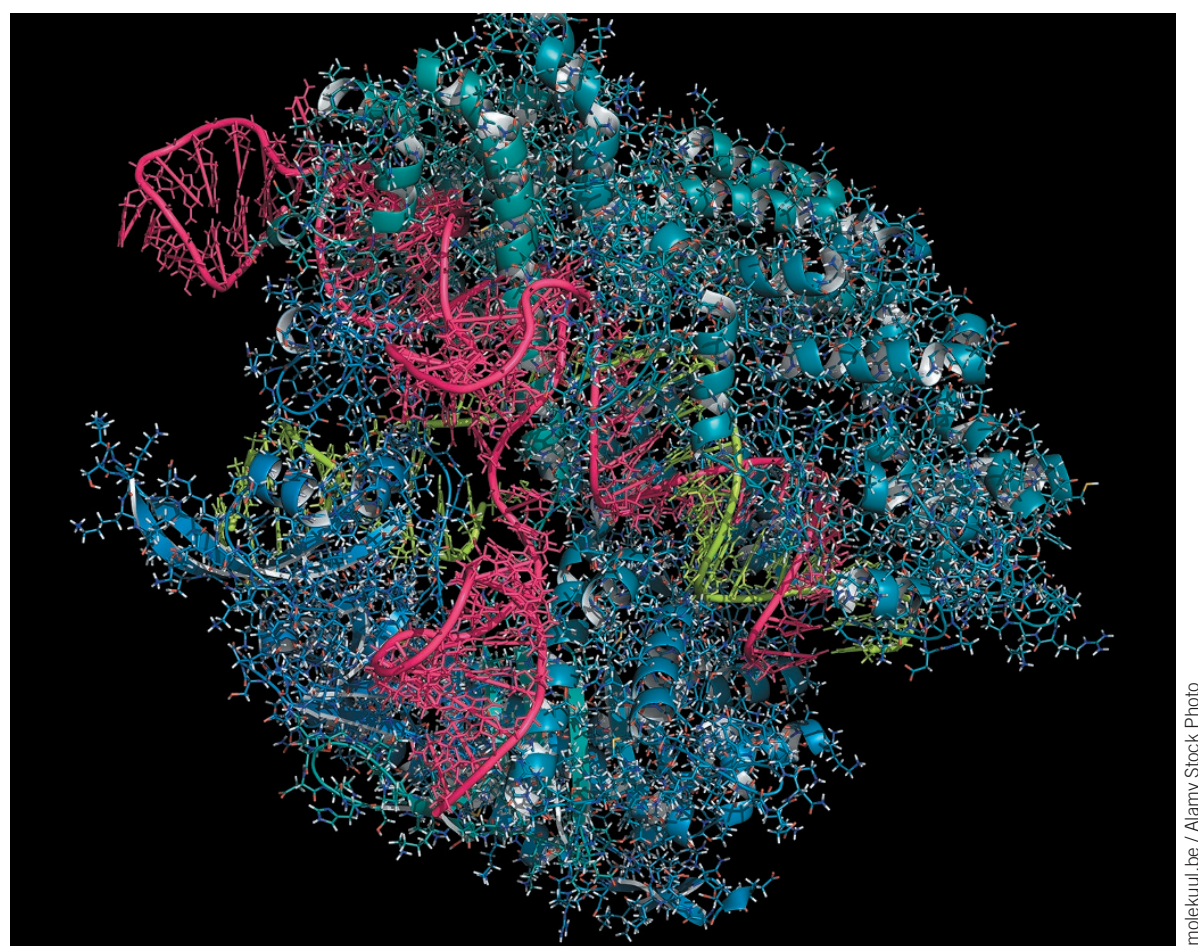

Cas9-the enzyme associated with CRISPR --plays a role in bacterial immunity.

Biohub, will accelerate research by cutting the lengthy wait associated with peer review. "This is a tipping point in biology," Quake told a crowd at Stanford's Big Data in Biomedicine Conference in May 2017. Biohub scientists are required to upload their papers to a preprint server before seeking traditional peer review. bioRxiv receives funding from the Chan Zuckerberg Initiative. As of early February, the Porteus and Weinberg preprint abstract had been viewed $~ 55,000$ times, and its PDF about 18,000 times.

Because CRISPR-Cas9 depends on a bacterial Cas9 enzyme its potential immunogenicity in humans, "was not an unappreciated aspect," says Harvard's Wagers. Porteus and Weinberg set out to uncover signs that the most widely used type of Cas9 proteins (from Staphylococcus aureus and Streptococcus pyogenes) elicited a reaction from the human immune system.

They looked at blood samples taken from 12 adults and also at the cord blood of 22 infants. They found that $79 \%$ of study participants had preexisting antibodies against $S$. aureus Cas 9 and $65 \%$ had antibodies against the enzyme from $S$. pyogenes.

In a related experiment, the researchers found that $46 \%$ of 13 adult volunteers produced T cells targeting Cas9 from $S$. aureus, though no T-cell responses against $S$. pyogenes Cas9 were detected.

"I really see this as more of a pilot project," says Thomas Boehm, a senior group leader at the Max Planck Institute of Immunobiology and Epigenetics in Freiburg, Germany. He describes the paper's publication as a "wakeup call," although the work did not show which, if any, functional consequences the findings may have in a real-life setting. Even so, "It's now in the world, it can be cited and it's causing confusion," he says. Wagers adds that some of the assay systems "may not be as robust as they could be."

Porteus acknowledges the limits of his research. For example, none of the antibodies have yet been cloned, nor were their binding affinities given against Cas9. "We don't think we've explored the issue completely. We've put a foot in the water; we hope labs that are more sophisticated than ours will take it on," he says. He is a scientific co-founder of CRISPR Therapeutics and holds stock in the company; he says the companies "obviously" weren't very happy with him.

Just a few weeks after news of the preprint broke, the three companies' stock had bounced back. More recently, it has been the overheating US economy, rather than preprints, that has poleaxed company stock prices.

Eva von Schaper Munich 